\title{
Quantitative surface normal measurement by a wavefront camera
}

\author{
Jian Ren, ${ }^{1, *}$ Xiquan Cui, ${ }^{1}$ Lap Man Lee, ${ }^{2}$ and Changhuei Yang ${ }^{1,2}$ \\ ${ }^{1}$ Department of Electrical Engineering, California Institute of Technology, 1200 East California Boulevard, Pasadena, California 91125, USA \\ ${ }^{2}$ Department of Bioengineering, California Institute of Technology, 1200 East California Boulevard, Pasadena, California 91125, USA \\ *Corresponding author: jren@caltech.edu
}

Received October 6, 2011; revised November 21, 2011; accepted November 22, 2011;

posted November 28, 2011 (Doc. ID 155731); published January 12, 2012

\begin{abstract}
A compact wavefront camera that allows users to quantitatively measure the intensity and wavefront at a remote object plane is reported. The camera is built from a chip-scale wavefront sensor that we previously developed. By measuring the wavefront of the image and calibrating the wavefront relationship between the image and object planes, the wavefront at the object plane can be computed and the surface normal of the object can be derived. We built a prototype camera and calibrated the wavefront relationship. In a proof-of-concept experiment, a set of concave mirrors with different focal lengths $(50-200 \mathrm{~mm})$, were imaged. The results agree well with their expected values. To demonstrate the application of the camera, we applied this method to measure the deformation of a microfluidic channel under pressure. @ 2012 Optical Society of America

OCIS codes: $\quad 010.7350,150.6044,120.0280,120.4630,110.5200$.
\end{abstract}

Wavefront sensing has been an active field of optical research over the past couple of decades and has been adapted for use in astronomy [1] and biomedicine [2]. Until recently, the relatively high cost and low effective pixel count associated with conventional wavefront sensors were significant obstacles that prevented their widespread usage. In recent years, various groups have focused on developing new forms of wavefront sensors [ 3 and 4]. Whether they are based on existing wavefront sensors or otherwise, imaging sensors have been designed for use in photographic camera context to achieve additional features [5] or the elimination of focusing optics [ㅁ]

In this Letter, we report a wavefront camera prototype that allows users to measure the wavefront and intensity maps of a remote object simultaneously. The wavefront sensing capability of this camera is obtained with the help of a wavefront imaging sensor (WIS) chip that we recently developed [7]. The WIS chip consists of a high pixel density complementary metal-oxide semiconductor (CMOS) chip that is coated with a spacer material and capped with a metal layer patterned with a grid of apertures. The aperture size and the spacer thickness are specially chosen for high Fresnel number operation so that light projected through the apertures onto the sensor can form well-focused and isolated light spots. By tracking the displacement of the light spots, we can then map out the phase gradient distribution of the incident light field. The WIS is distinct from other wavefront sensing technologies in that it is simple and easily manufacturable via a standard CMOS sensor foundry, and that its effective pixel density is remarkably high (the WIS prototype has an effective pixel pitch of $11 \mu \mathrm{m}$ ).

By placing this sensor in the image plane of a photographic camera, we will be able to collect both an intensity image and a phase gradient (or wavefront) image of the target object. Specifically, we note that the wavefront at the image plane is related to that at the object plane. The relationship depends on the optical configuration of the imaging system and can be experimentally acquired by a calibration procedure. Upon obtaining this quantitative relationship of the wavefronts, one can convert the measurement results on the image plane to the object plane. Therefore, with a single snapshot, the camera can capture the wavefront of a remote object while taking a common intensity image at the same time, as traditional cameras usually do. Furthermore, one can deduce the object surface normal from the wavefront map.

Based on the above idea, we built a prototype camera. It includes two major parts: the WIS chip and the camera system. The sensor chip has the same structure and specification as the ones used in [7]. As shown in Fig. 1(a), it contains a 280 by 350 matrix of apertures on a $150 \mathrm{~nm}$ thick aluminum layer. The apertures have a nominal diameter of $6 \mu \mathrm{m}$. The spacing between adjacent apertures is $11 \mu \mathrm{m}$, which allows a 5 by 5 sensor pixel matrix with a pixel size of $2.2 \mu \mathrm{m}$ to be allocated to each aperture. The sensor chip and its supporting input/output electronics are enclosed in a metal case. An Ethernet cable is used to transfer acquired data to a computer. A Format 1 closed-circuit television lens (Pentax Model B5014A) was selected for the camera system. It has a focal length of $50 \mathrm{~mm}$ and an $f$-number of 1.4. A customized bellow adapter was designed to mount the lens onto the enclosure of the wavefront sensor chip. The adaptor can extend the standard flange back length of the $\mathrm{C}$ mount $(17.526 \mathrm{~mm})$, by about $20 \mathrm{~mm}$ maximally. By placing the sensor farther away from the lens, the adaptor essentially shortens the nearest object distance and thus increases the magnification. The maximal magnification has been increased from the standard value of 0.05 to 0.4. This enabled us to take close-up photographs for
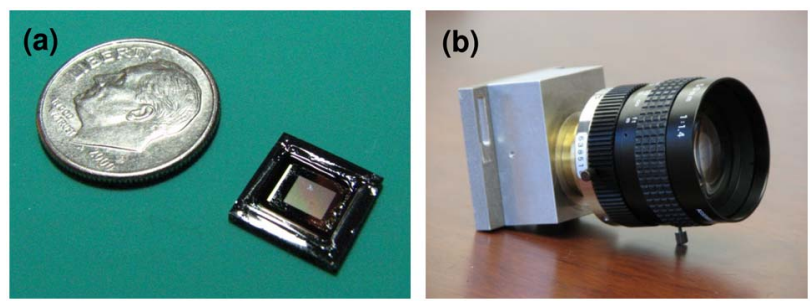

Fig. 1. (Color online) Photographs of (a) the wavefront sensor chip with a U.S. dime and (b) the prototype wavefront camera. 
detailed surface analysis. A photograph of the prototype wavefront camera used in this work is displayed in Fig. 1(b).

As the wavefront sensor measures the local phase gradients of the light field in close proximity to its top surface, a relationship between the wavefronts of the image plane and the object plane needs to be established. To analyze this relationship, we first look at the wavefront sensor at the image plane. Each image pixel unit (IPU) is composed of the aperture and its sensor pixel matrix underneath [7]. It measures the light projection through the aperture. This light distribution represents the angular spectrum of the local light field and the IPU essentially works as a tiny angular spectrum analyzer. Notice that the light field around a sample point at object plane $U_{o}(x, y)$ and that around its corresponding image point at image plane $U_{i}(x, y)$ of a perfect imaging system can be related to each other as the following []]:

$$
U_{i}(x, y)=\frac{1}{|M|} U_{o}\left(\frac{x}{M}, \frac{y}{M}\right)
$$

where $M$ is the system's magnification. Because the local angular spectrum $A\left(k_{x}, k_{y}\right)$ is actually a Fourier transformation of the local light field, the relationship of the local angular spectrum at the image point $A_{i}\left(k_{x}, k_{y}\right)$ and that of the object point $A_{o}\left(k_{x}, k_{y}\right)$ can be derived as follows:

$$
A_{i}\left(k_{x}, k_{y}\right)=|M| A_{o}\left(M k_{x}, M k_{y}\right),
$$

where $k_{x}$ and $k_{y}$ are the spatial frequencies. When light is reflected from a sample location at the object plane by an object and a reference surface, respectively, the relationship between the corresponding local angular spectrums $A_{i s}\left(k_{x}, k_{y}\right)$ and $A_{i r}\left(k_{x}, k_{y}\right)$, at its image location, can be expressed as:

$$
\begin{aligned}
A_{i s}\left(k_{x}, k_{y}\right) & =A_{i r}\left(k_{x}-\frac{\delta k_{x}}{M}, k_{y}-\frac{\delta k_{y}}{M}\right), \delta k_{x}=2 k \theta_{x}, \delta k_{y} \\
& =2 k \theta_{y} ; \quad \text { while } \quad \delta k_{x}, \delta k_{x} \ll k,
\end{aligned}
$$

where $k$ is the wavenumber of the light wave, and $\theta_{x}$ and $\theta_{y}$ represent the surface normal difference between the reference and object surface at the sample location on the object plane. In case of demagnification $(|M|<1)$, Eq. (3) indicates that the shift of the angular spectrum at the image plane is magnified $1 /|M|$ times relative to the shift at the object plane. The spectrum stretching is a direct result of Eq. (2).

In the practical operation of the wavefront sensor, the centroid of the light distribution on the sensor pixel matrix has been abstracted to represent the phase gradient - the direction of the local wavefront. This procedure simplifies the local angular spectrum to a single $\delta$ function (valid as long as the bandwidth of the local angular spectra is narrower than the acceptable bandwidth of the camera). For these cases, the shift of the centroid will also be magnified $1 /|M|$ times if the sensor was brought from the object plane to the image plane. Therefore, the object surface normal difference from a reference sur- face can be assessed by measuring the wavefront change at the image plane - the centroid shift.

We can expect practical systems to deviate in their adherence to these relationships due to system aberrations. To compensate these effects, we experimentally calibrated the relationship between the object surface normal and the wavefront map at the image plane. First, the camera system was configured at a magnification of 0.223 . A plane mirror was used as the object, whose surface normal is uniform across the entire plane. During the calibration, the mirror was tilted to reflect the collimated illumination white light both horizontally and vertically. While it was scanned over a range of the surface normal direction, the centroid displacement map was calculated and recorded for each scan angle. Figure 2 summarizes the results of the horizontal scanning, and those of the vertical scanning are analogous. A typical plot of the centroid displacement at an IPU is shown in Fig. 2(a), where zero tilt angle refers to the plane perpendicular to the main axis of the lens optics. The red dashed curve shows that the measured shift of the centroid is linear to the scan angle. Deviations on both ends of the curve are because an image point was not formed at the IPU for those scan angles due to the vignetting of the system. To record this relationship, a linear fit at a least squares sense was used to abstract the slope and the offset. As the centroid displacements were measured at two dimensions, both slope and offset have two components. Figures 2(b) and 2(c) are the down-sampled vector representation of the slope and the offset across the entire sensor surface. Figure 2(b) has a nonuniform distribution due to the lensing and the aberrations of the camera system. In contrast, the slope plot in Fig. 2(c) shows a fairly uniform distribution. This indicates that, no matter how the wavefronts at zero scan angle are different for each IPU, their wavefront changes (centroid shift) upon certain surface normal change would be more or less the same. This is a natural consequence of Eq. (3).

Based on the measured slopes, one can estimate the system magnification. The mean of the slopes was about $-0.74 \mathrm{pixel} /$ degree. And the relationship between the centroid shift and the local wavefront change had been

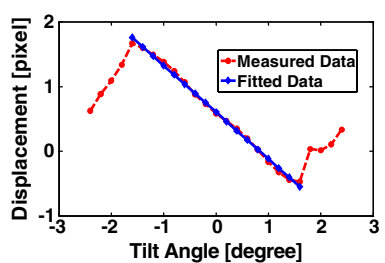

(a)

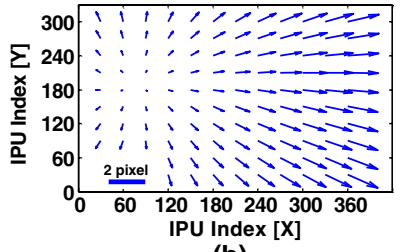

(b) (c)

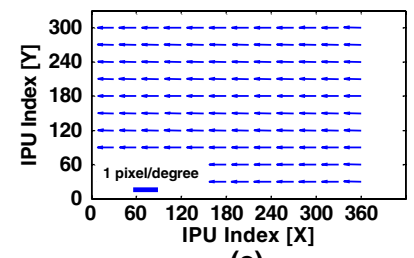

Fig. 2. (Color online) Wavefront calibration: (a) the relationship between the scan angle and the centroid displacement, (b) the vector map of the fitting parameter-the offset, and (c) the vector map of the fitting parameter-the slope. 


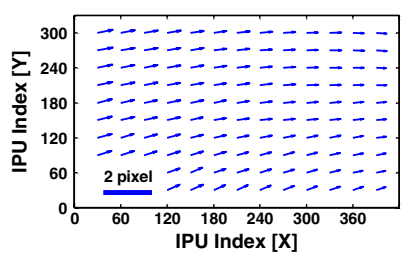

(a) (b)
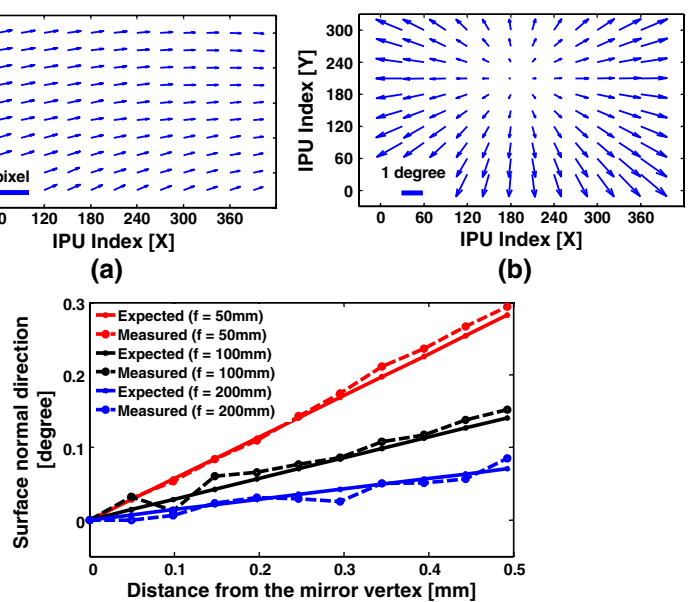

(c)

Fig. 3. (Color online) Validation experiments: (a) the vector map of the measured wavefront (centroid displacement) for a $200 \mathrm{~mm}$ concave mirror, (b) the vector map of the surface normal deduced from (a), and (c) the comparison of the measured and the expected surface normal profiles (along the horizontal direction).

previously measured to be about $11.95 \%$ pixel. These values yield an average ratio of $4.42(=(11.95 \times 0.74) / 2)$ between the shifts of the angular spectrum at the image and the object planes. Using Eq. (3), we can estimate the system magnification as $0.226(=1 / 4.42)$, which agrees well with the measured value 0.223 .

Next, to validate this method, we selected a series of spherical concave mirrors $(f=50,100,200 \mathrm{~mm})$, as objects with known surface shape, to be measured by the prototype camera. We brought the concave mirrors into the focus of the camera by placing them at an object distance of $25.7 \mathrm{~cm}$ from the front edge of the camera lens. After the objects were placed in focus, we snapped images for each mirror. The acquired images were first analyzed to acquire the wavefront map at the image plane and then converted to the wavefront map of the object plane using the known parameters (offset and slope) acquired in the calibration procedure. Finally, we deduced the surface shape information (surface normal) of the objects.

For the $200 \mathrm{~mm}$ concave mirror, the vector map of the centroid displacement at the image plane is shown in Fig. 3(a). The deduced surface normal map of the object is shown in Fig. 3(b). In comparing Figs. 3(a) and (b), we can appreciate the calibration and the conversion procedures, as they enable us to transform the measured wavefront on the sensor to the surface normal of a remote object. Finally, Fig. 3(c) is the comparison between the surface normal (horizontal) profiles measured by this method and those based on the mirror specifications. These profiles agree with each other, which validates the method.

To demonstrate the application of this device, we used the prototype wavefront camera to examine the surface of a microfluidic chip. As shown in Fig. 4(a), the chip under study has a cross-bar-shaped channel with a nominal width of $0.5 \mathrm{~mm}$ and an inlet and outlet pair. By adjusting the flow speed at the inlet and the outlet, the fluid pressure inside the channel can be controlled. Because of the flexibility of polydimethylsiloxane, the material of the channel membrane, the fluid inside will generate deformation and
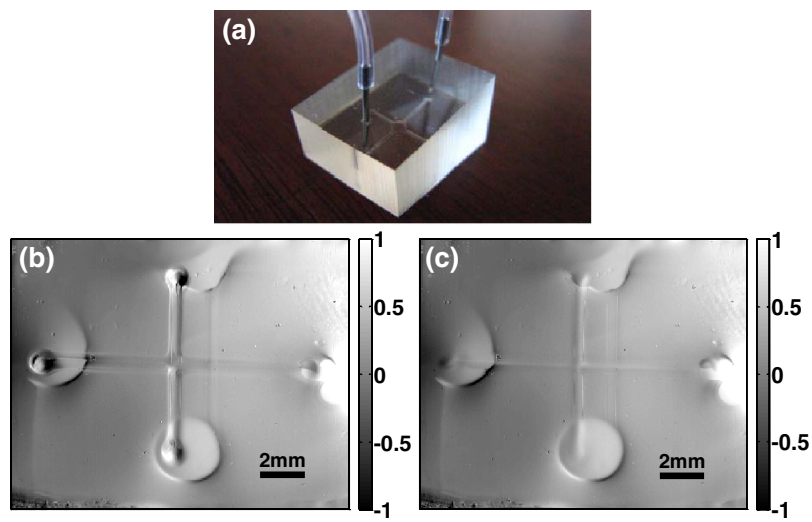

Fig. 4. (Color online) Deformation of a microfluidic channel: (a) the photography of the microfluidic chip, (b) the horizontal direction surface normal of the channel under high pressure, and (c) the horizontal direction surface normal of the channel under low pressure. The unit of the color bars is degree.

create nonuniform curvature on the top surface depending on the internal pressure. To quantitatively measure this deformation, we imaged the top surface by the prototype camera while the channel was under high and low pressure. The resulting surface normal (horizontal direction) maps are displayed in Figs. 4(b) and (c) respectively. As those maps are rendered at the same color scale, one can clearly tell the surface under high pressure had larger deformation than that under low pressure. The quantitative curvature value of any point of interest on the surface can be computed, as the maps show the angles of surface normal. Because the deformation is a function of the internal pressure, this camera provides a simple way to monitor the internal pressure quantitatively, remotely, and very likely in real time, as well.

When converting a traditional photographic camera to a wavefront camera, the required hardware modification is minimal-basically only the sensor chip needs to be upgraded to the WIS. Therefore, the wavefront camera delivers an easy, cost-effective, and quantitative solution for everyday wavefront measurement demands.

The authors acknowledge Jigang Wu, George Rakuljic, and Guoan Zheng for helpful discussion. This work was supported by the Wallace Coulter Foundation, National Science Foundation (NSF) career award BES-0547657, and the National Institutes of Health (NIH) R21EB008867.

\section{References}

1. R. W. Wilson and C. R. Jenkins, Mon. Not. R. Astron. Soc. 278, 39 (1996).

2. T. Salmon, L. Thibos, and A. Bradley, J. Opt. Soc. Am. A 15, 2457 (1998).

3. J. Primot and L. Sogno, J. Opt. Soc. Am. A 12, 2679 (1995).

4. R. Cudney, Opt. Express 19, 17396 (2011).

5. R. Ng, M. Leovy, M. Bredif, G. Duval, M. Horowitz, and P. Hanrahan, "Light field photography with a hand-held plenoptic camera," Stanford Tech. Report CTSR 2005-02 (Stanford University, 2005).

6. P. Gill, C. Lee, D. Lee, A. Wang, and A. Molnar, Opt. Lett. 36, 2949 (2011).

7. X. Cui, J. Ren, G. Tearney, and C. Yang, Opt. Express 18, 16685 (2010).

8. J. W. Goodman, Introduction to Fourier Optics, 3rd ed. (Roberts, 2004). 